\section{Otopathic voices and alcoholic visions}

Sir - In the March 1998 issue there were two apparently unconnected papers with no common references. One was on voices associated with otitis externa, ${ }^{1}$ the other on visions during alcohol withdrawal. ${ }^{2}$ Inspection of references missing from these papers suggests that all these hallucinations have an otopathic basis, or at the very least, a common specific physiological mechanism. Carroll and Milnes ${ }^{1}$ write "Associations between peripheral sensory organ disease and hallucinations in the corresponding modality are well recognised. A relationship between psychiatric illness and ear disease has been noted since the turn of the century". In fact, there is an extensive literature from over 100 years ago, still considerably in advance of modern writings on this topic. ${ }^{3-7}$ Many have reported that for ear disease hallucinations were not confined to the same modality, and could be visual. . $^{3,4,6,8}$

They ${ }^{1}$ could also not find any previous otopathic case with passivity symptoms. Jaspers ${ }^{y}$ gives four of the cardinal symptoms of Meniere's disease as examples of passivity phenomena (tinnitus, giddiness, audiosensitivity, pressure in the head). Without systematic searching, many old cases can be found. Margery Kempe, ${ }^{7}$ the 15 th century mystic, had Meniere's disease with tinnitus which she said turned into birdsong under God's influence. Both Joan of Arc and $\mathrm{R}$ Schumann had Meniere's disease with visions of angels supposedly influencing their auditory hallucinations. Martin Luther ${ }^{6,7}$ also had Meniere's disease with tinnitus changing into a musical; in his case, he blamed the devil for causing his ear disease. He also had otorrhea, possibly from otitis externa, and visual hallucinations. A nun with long-standing ear infections and deafness ${ }^{3}$ was tormented by voices which she thought the abbess was communicating to her via a phonograph. A woman (Norman 1902) had tinnitus in her deaf right ear attributed to electrical machines; she also heard dextral voices, blasphemous at times, which were directing these machines; her first psychiatric symptom had been a vision of an angel in church. A second patient with non-infective otitis heard voices in deaf right ear; he had exquisite delusions of occult influence and passivity phenomena resembling those in witchcraft, and had visual hallucinations on closing his eyes. Houston and Royce briefly described two patients: one denied she was deaf but obviously was very deaf, and had delusions that sounds and voices were travelling up a water pipe; a man with otitis heard banging and tapping noises, sirens and voices, and thought that somebody was putting their thoughts into his head. A man " with left whistling tinnitus, attributed to a surveillance device, heard voices in his deaf left ear; an alcoholic woman with persistent deafness, buzzing and a voice in her left ear displayed passivity phenomena. An otosclerotic" was convinced that voices were able to read his thoughts, and that someone was putting microphones in his room. Curiously, there is a striking religious content in many of these cases.

Carroll and Milnes state that the association between ear disease and mental illness was considered by early workers to be due to infective processes in the ear affecting the brain directly: I listed ${ }^{5} 10$ very distinguished clinicians from the last century who dismissed this contiguity theory. They also cited a recent case-control study reviving this theory; in my reply to Mason and Winton, I gave 11 reasons for doubting the mediation of temporal lobe irritation, the most conclusive being that unilateral hallucinations are ipsilateral to the ear disorder. ${ }^{1,6,11}$ If from the brain, they should be heard in the opposite healthy ear. Actually, any infective mechanism is untenable since there are many examples of auditory hallucinations in non-infective ear disease.

Sloan and O'Boyle ${ }^{2}$ were unable to find any reference to a previous case of alcoholic hallucinosis presenting with visual hallucinations only. An alcoholic ${ }^{12}$ hallucinated vivid landscapes without auditory accompaniment; the trees sometimes appeared upside-down or sideways, leading Schilder to infer a vestibular origin. Gross et al ${ }^{13}$ intensively studied 500 patients with acute alcoholic psychosis in order to elucidate hallucinogenic mechanisms. Visual hallucinations usually began after auditory ones, but they occurred alone in $11 \%$ of patients. Hallucinations in general correlated 0.48 with clouding of the sensorium but 0.62 with tinnitus, showing that tinnitus was the significant hallucinogenic factor. Whatever the mechanism it was more closely related to auditory than visual hallucinations, which were secondary or more pathological features. Tinnitus in alcohol withdrawal is of peripheral origin: 14 visual hallucinations appeared in $77 \%$ of cases and unstructured visual perceptions such as shadows, spots, lights and flashes in $57 \%$. A likely source for these is the vestibular system, as was indeed implicated in their patient, ${ }^{2}$ who was nauseous and retched (no mention was made of checks for other symptoms of inner ear hyperirritability-audiosensitivity, tinnitus, fullness, vertigo, deafness). In labyrinthine fistulas Schiler ${ }^{12}$ noted narrowing of the visual field, darkening, small glittering circles and scintillation in the optic field; experimental activation of the vestibular labyrinth has induced complex disturbances, including misting, diplopia, multiplication of animals or persons, micropsia, metamorphosis, eidetic images and a vision of the sinking of the Titanic! He has other detailed descriptions of visual hallucinations in alcoholic and other intoxications, and supplied a mass of evidence to back his view that these were usually vestibulogenic. Haloperidol alleviated the visual hallucinations, indicating a common mechanism with auditory hallucinations in schizophrenia, ${ }^{\circ}$ eg. inner ear activation. Other cases show a strong interplay between otopathic and alcoholic hallucinations. In the $1937 \mathrm{case}^{2}$ of alcoholic auditory and visual hallucinations, the tinnitus, music and voices were clearly otopathic, being heard only on the side of an old right otitis. Bechterew ${ }^{3}$ reported that otopathic hallucinations were exacerbated by alcohol. Toulouse in $1892^{6}$ reported four cases of unilateral auditory hallucinations allegedly of cortical origin: however, at least three had ear disease, one had voices in his deaf ear aggravated by alcohol and two others were alcoholic wine merchants! The otopathic psychosis of Norman's second patient ${ }^{5}$ started with a bout of drunkenness resulting in imprisonment. An otosclerotic ${ }^{11}$ twice had vivid visual hallucinations during alcohol withdrawal. After these resolved the first time, his tinnitus transformed into music; after the second, the music changed into voices.

Summarising, some bizarre or unlikely facts are evident: 1. Otitis media predisposes to schizophrenia.

2. Episodic auditory hallucinations are prominent in schizophrenia. 


\section{A most effective}

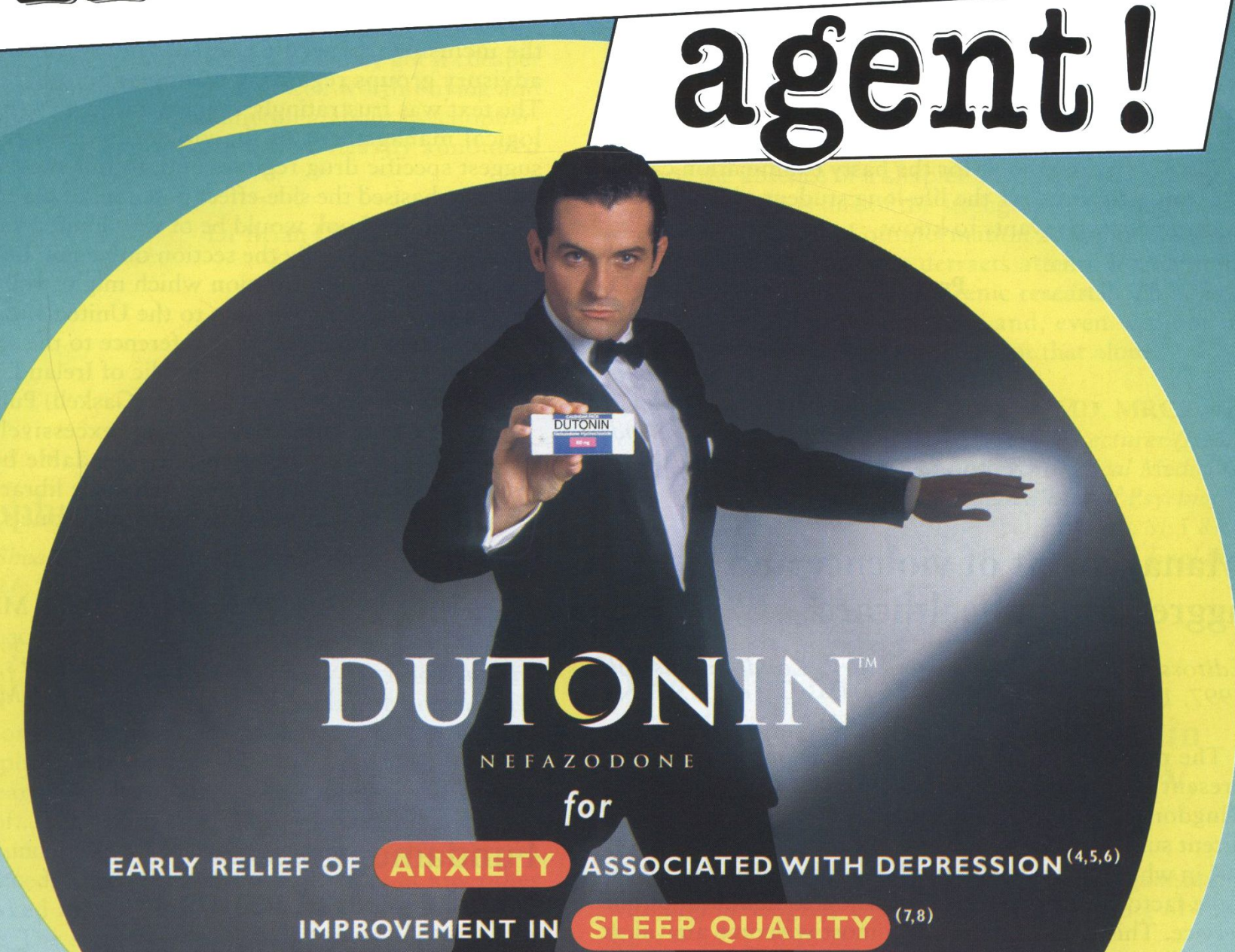

IMPROVEMENT IN SLEEP QUALITY ${ }^{(7,8)}$

WITHOUT COMPROMISING SEXUAL FUNCTION (1)

FIRST-LINE IN TREATING DEPRESSIO N ${ }^{(1,2,3)}$

Product Authorisation Numbers: Dutonin "' Tablets 50mg P.A. 2/60/1. Dutonin"' Tablets 100 mg P.A. 2160/2. Dutonin "'T Tablets 200mg P. 2/60/3.

Holder: BristolMyers Satb Phe

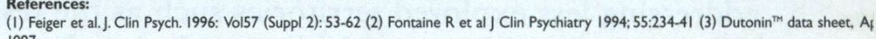

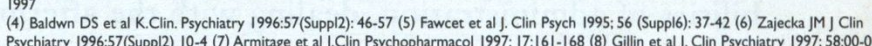
(9) MIMS, November 1997

Bristol-Myers Squibb Pharmaceuticals Limited 
3. Serous ${ }^{5}$ or purulent otitis media can trigger polysensory hallucinations.

4. Diseased ears are oversensitive to $\mathrm{drugs}^{8,13}$ and alcohol amplifies existing otopathic hallucinations.

5. Auditory hallucinations in alcoholics arise from peripheral tinnitus.

6. Visual and auditory alcoholic hallucinations have common correlates.

7. Hallucinations and some psychotic symptoms ${ }^{7}$ arise from irritation of the peripheral audiovestibular system.

The last conclusion is the most bizarre of all, but has the considerable merit of removing the need for six other feeble, ad hoc, or desperate counter-explanations.

AG Gordon

32 Love Walk,

London SE5 8AD, England.

References

1. Carroll A, Milnes D. Unilateral auditory hallucinations in association with ear infection. Ir J Psych Med 1998; 15:31-2.

2. Sloan D, O’Boyle J. Visual alcoholic hallucinosis. Ir J Psych Med 1998; 15: 35-6.

3. Bechterew. On hallucinatory insanity following affections of the ear. J Ment Sc $1904 ; 50: 169-71$.

4. Gordon AG. Unilateral auditory hallucinations. Br J Psychiatry 1988; 153 263-4.

5. Gordon AG. Ear disease and schizophrenia - brain not needed? Acta Psychiatr Scand 1996: 93: 409-10.

6. Gordon AG. Unilateral auditory hallucinations: ear or brain? 1997; 63: 814. 6. Gordon AG. Unilateral auditory hallucinations: ear or bran? $1997 ; 63: 814$
7. Gordon AG. Insight into auditory hallucinations and psychosis. Int J Ger Psychiatry 1997; 12:410-1.

8. Gordon AG. Benzodiazepines and the ear - tinnitus, hallucinations and schizophrenia. Can J Psychiatry 1993; 38: 156-7.

9. Jaspers K. General psychopathology. Manchester: Manchester University Press, 1963.

10. McBride A, Hamilton-Kirkwood L. Unilateral auditory hallucinations. $\mathrm{Br}$ J Psychiatry 1988; 153: 264.

11. Marneros A, Beyenburg St, Berghaus A. Unilateral hallucinations and other psychotic symptoms due to otosclerosis. Psychopathology 1997; 30: 89-92.

12. Schilder P. The vestibular apparatus in neurosis and psychosis. ] Nerv Ment Dis $1933 ; 78: 1-23,137-64$

13. Gross MM, Halpert E, Sabot L. Toward a revised classification of the acute alcoholic psychoses. J Nerv Ment Dis 1967; 145: 500-8.

14. Saraway SM, Pardes H. Auditory elementary hallucinations in alcohol withdrawal psychosis. Arch Gen Psychiatry 1967; 16:653-8.

\section{Alcoholic hallucinosis revisited}

Sir - We thank Dr Gordon for his interesting comments on our case report. Firstly he quotes two previous references from the literature (Schilder 1933,' Gross 1967²) on the isolated occurrence of visual hallucination in alcoholic psychotic states, secondly he suggests that the visual hallucinations in our patient may be due to ear disease specifically vestibular dysfunction. Our literature search did not reveal the $1933^{\prime}$ and $1967^{2}$ references and we are grateful to Dr Gordon and his clearly in-depth knowledge of this topic for providing us with these references. We described the presentation of our case as "unusual in that the hallucinations described were in the visual modality only". The fact that these two previous reports date back some 65 and 31 years respectively may therefore support our contention that this presentation is unusual if not extremely rare. Fortunately we were able to obtain copies of both references. We would however have concerns firstly regarding the description by Schilder et al of 'an alcoholic with emotional deterioration' as we are not given any further information about whether he had continued to drink alcohol, whether he had ceased alcohol intake, whether he was in withdrawal or indeed would fulfill criteria for 'alcoholic hallucinosis'. Similarly we would express our concern regarding the cohort of patients described by Gross et al, specifically their finding that "hallucination in general correlated 0.48 clouding of the sensorium". Using ICD $-10^{3}$ clear consciousness is required to diagnose alcoholic hallucinosis which comes under the rubric "alcohol induced psychotic states predominantly hallucinatory" and it is not clear therefore that the cases considered by Gross satisfy modern criteria for alcoholic hallucinosis as such the cases cited above may not therefore be comparable to our individual case.

Regarding the second point Dr Gordon provides an interesting alternative explanation for the genesis of visual hallucinations in alcoholic hallucinosis citing additional case reports and references. In the case of our patient, there was no clinical evidence of vestibular dysfunction. The transient early morning retching could have represented such a symptom but had been present through much of the patients long 'drinking-life' and was much more likely to be a symptom of alcohol withdrawal. We cited a number of recent references detailing neurotransmitter dysfunction specifically hyperdopaminergic states in alcohol withdrawal which would support the development of visual hallucinations in our case. Finally, may we restate the fact that controversy surrounding the nature of alcoholic hallucinosis has been present in the literature since its first description in $1847 .{ }^{4}$

Darina Sloan, MB, MRCPSych, Senior Registrar, University College Hospital, Galway, Ireland. James O'Boyle, MB, FRCPsych, Consultant Psychiatrist,

St. Vincent's Hospital, Fairview, Dublin 3, Ireland.

\section{References}

1. Schilder P. The vestibular apparatus in neurosis and psychosis. J Nerv Ment Dis $1933 ; 78: 1-23,137-64$.

2. Gross MM, Halpert E, Sabot L. Toward a revised classification of the acute alcoholic psychosis. Arch Gen Psychiatry 1967; 16:652-8.

3. World Health Organisation. The $1 C D-10$ classification of mental and behavioural disorders, clinical descriptions and diagnostic guidelines. Geneva: WHO, 1992.

4. Marcel CNS de la folie causer par l'abus des besissiens alconliques. Thesis (Paris Rignoux, imprimeur de las faculte de medecin, 1847).

\section{Re: Unilateral auditory hallucinations}

Sir- I would like to thank Dr Gordon for bringing to our attention several cases similar to that which we reported. Over the three years which have passed since the patient in question was first admitted to hospital, it has become clear that the diagnosis is paranoid schizophrenia. The most parsimonious explanation for the symptomatology described is simply that the ear infection which coincided with the first psychotic episode exerted a pathoplastic effect on the symptomatology. Dr Gordon does not appear to have considered this possibility in the list of "bizarre or unlikely facts" which concludes his letter.

Andrew Carroll MRCPsych, Research Fellow Department of Psychiatry, The University of Edinburgh, Royal Edinburgh Hospital, Morningside Park, Edinburgh EH10 5HF, Scotland. 